\title{
Vibrating Reed Study of Superconducting Cuprates Fabricated by Superfast Melt Quenching in a Solar Furnace
}

\author{
J. Chigvinadze \\ V. Tavkhelidze \\ G. I. Mamniashvili \\ Department of Condensed Matter Physics, Department of Condensed Matter Physics, Department of Condensed Matter Physics, \\ Andronikashvili Institute of Physics, \\ Ivane Javakhishvili Tbilisi State \\ University,Tbilisi, Georgia \\ chigvinadze@yahoo.com \\ Andronikashvili Institute of Physics, \\ Ivane Javakhishvili Tbilisi State \\ University,Tbilisi, Georgia \\ tawge@yahoo.com \\ Andronikashvili Institute of Physics, \\ Ivane Javakhishvili Tbilisi State \\ University,Tbilisi, Georgia \\ mgrigor@rocketmail.com

\section{G. Donadze} \\ Department of Condensed Matter Physics, \\ Andronikashvili Institute of Physics, \\ Ivane Javakhishvili Tbilisi State \\ University,Tbilisi, Georgia \\ donadze_g@yahoo.com

\section{J. V. Acrivos} \\ Department of Chemistry, \\ San Jose State University, \\ San Jose, USA \\ jacrivos@earthlink.net \\ D. Gulamova \\ Institute of Materials Science SPA \\ "Physics-Sun" of Academy of Science, \\ Tashkent, Uzbekistan \\ gulamova@uzsci.net
}

\begin{abstract}
In this study, the potential possibilities of the precision of the vibrating reed method for the evaluation of the $T_{c}$ of superconducting precursors in an HTSC Bi-Pb-Sr-Cu-O system were investigated. A special technology for obtaining these samples by using solar energy for melting and following superfast melt quenching was applied to increase their internal inhomogeneity, allowing to receive high-quality textural ceramic samples of $\mathrm{Bi}_{1,7} \mathrm{~Pb}_{0,3} \mathrm{Sr}_{2} \mathrm{Ca}_{n-1} \mathrm{Cu}_{n} \mathrm{O}_{\mathrm{y}} \quad(\mathrm{n}=2-30)$ showing critical temperatures of superconducting precursor transitions above bulk $T_{\mathrm{c}}$. To determine critical temperatures of superconducting precursors above bulk $T_{\mathrm{c}}$ for the first time, the original vibrating reed method of studying these multiphase samples in an applied magnetic field was used. It was shown that this method has sensitivity to superconducting diamagnetism making possible to reveal new superconducting precursor phases above bulk $T_{c}$.
\end{abstract}

Keywords-HTSC; solar energy; superfast quenching; vibrating reed technique; ctitical temperature of superconducting transition; high-temperature superconducting phases; pinning

\section{INTRODUCTION}

After the discovery of high-temperature superconductors (HTSC) in 1986, great efforts were made to the further increase the critical temperature of superconducting transition $T_{c}$ with aim to reach room temperatures. Such a discovery would cause the emergence of new HTSC materials with $T_{\mathrm{c}}$ higher than the ones of liquid nitrogen $(\sim 77 \mathrm{~K})$ and hydrogen $(19 \mathrm{~K})\left(T_{\mathrm{c}}\right.$ in $\mathrm{YBaCuO}$ and $\mathrm{MgB}_{2}$, correspondingly) opening great possibilities for their applications in electronics and energetics. From this point of view, particular interest attracts the $\mathrm{Bi}-\mathrm{Pb}$ $\mathrm{Sr}-\mathrm{Ca}-\mathrm{Cu}-\mathrm{O}$ system, because it is characterized by high $T_{\mathrm{c}}=107 \mathrm{~K}$ and the record high second critical magnetic field $H_{\mathrm{c} 2}$ $\sim 150 \mathrm{~T}$. Our group has undertaken research on the possibility to increase the $T_{\mathrm{c}}$ of this system [1-5]. In [1-3, 5] the melt technology for obtaining $\mathrm{Bi}-\mathrm{Pb}-\mathrm{Sr}-\mathrm{Cu}-\mathrm{O}$ HTSC ceramics using solar energy for melting and subsequent superfast melt quenching for increasing of internal inhomogeneities was developed, allowing the receiving of homogeneous, highquality, textured ceramic samples with controllable microstructure and pinning centers. In [4], Hot Shock Wave Consolidation Technology was used to produce $\mathrm{Bi}-\mathrm{Pb}-\mathrm{Sr}-\mathrm{Ca}-$ $\mathrm{Cu}-\mathrm{O}$ compositions with increased internal inhomogeneity and for studying their superconducting precursor phases by torsional magnetometry method. This time, for increasing internal inhomogeneity we used solar melting and following superfast quenching technology.

Previous measurements of pinning and dissipation processes were carried out in the Andronikashvili Institute of Physics using an original vibrating torsion mechanical magnetometry method to study the torsion oscillations of samples in an applied magnetic field realized using an automated multipurpose device [6] and standard methods of magnetic and electric parameter measurements. It was showed that samples obtained using the suggested solar technology were multiphase containing phases with higher critical temperatures $T_{\mathrm{c}}$ reaching $\sim 200 \mathrm{~K}$. But the concentration of new HTSC phases appeared to be so small that for more precision assessments it was necessary to find alternative and more sensitive methods. One of such methods could be the vibration reed (VR) method for the first time developed in works of our group and then independently [7]. As the frequency and dissipation of VR could be measured with high precision, the VR method exceeds in sensitivity a conventional ac- 
susceptometer, and besides, it is contactless. Using the VR method one could study the elastic coupling between the Abrikosov vortex lattice and the crystal lattice even in micron sized grains inserted in the normal matrix. The field dependence measurements of VR resonance frequency and dissipation could be used for the precision investigation of the first critical magnetic field $H_{\mathrm{c} 1}$ temperature dependence without the introduction of free parameters, along with the energy distribution character caused by the motion of the Abrikosov vortex lattice.

In [8], the example of successful application of the VR method for investigation of two-phase $\mathrm{Bi}-\mathrm{Ca}-\mathrm{Sr}-\mathrm{Cu}-\mathrm{O}$ superconducting ceramics and the precision assessment of their bulk critical temperatures $T_{\mathrm{c}}$ were presented. The VR method was used for the first time to determine critical temperatures of superconducting precursors above bulk $T_{\mathrm{c}}$. So, the aim of the current work is to determine critical temperatures of superconducting precursors above bulk $T_{\mathrm{c}}$. We used for the first time the original VR method to study these multiphase samples in an applied magnetic field. It was shown that this method has sensitivity to superconducting diamagnetism sufficient to reveal new superconducting precursor phases above bulk $T_{\mathrm{c}}$.

\section{RESULTS AND DISCUSSION}

For the study of high-temperature superconductors, we used a setup consisting of a cryostat with a block-holder of the sample, placed in the gap of a permanent magnet, and an electronic part. The electronic part of the setup contains a mechanical spectrometer developed in the Andronikashvili Institute of Physics operating at a frequency of $\sim 1 \mathrm{kHz}$ [9], and instruments for powering the permanent magnet and creating a weak $(\sim 0.5 \mathrm{mT})$ alternating magnetic field on the sample. The high sensitivity of the equipment provides measurements of the natural frequency of the sample $f$ with an accuracy of $\sim 0.1 \%$. Figure 1 shows actual devices/set up view and measuring cell photos. Figure 2 shows the principal electronic circuit of the installation.

(a)

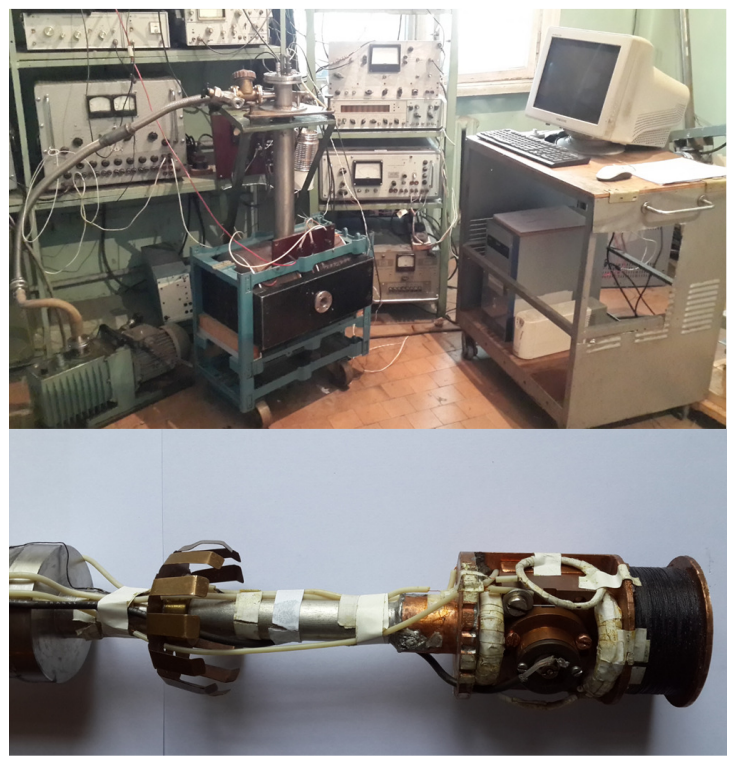

Fig. 1. (a) Actual devices/set up photo, (b) measuring cell.

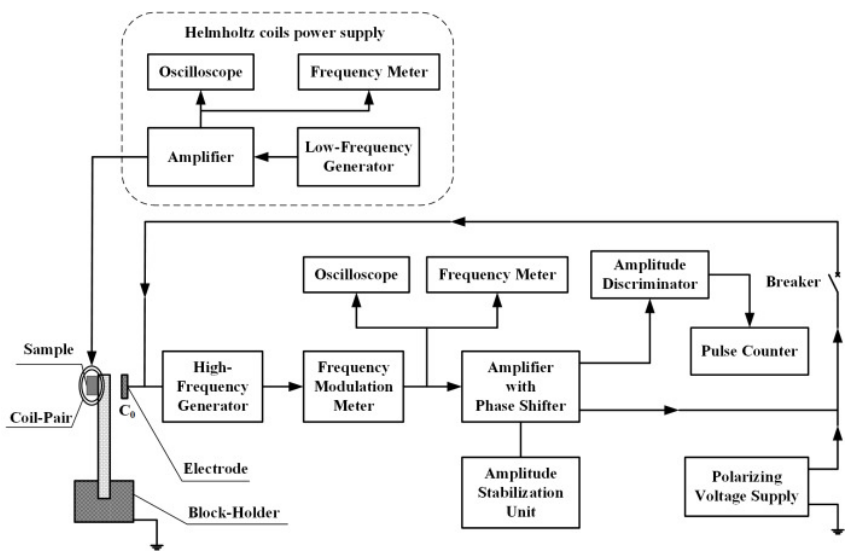

Fig. 2. Electronic block-diagram of the installation.

The basis of the operation of a mechanical spectrometer is the electrostatic method of exciting bending oscillations of a sample having the shape of a rectangular plate. The electronic equipment of the spectrometer allows measurements in the mode of self-excitation of samples at their natural resonant frequency. The electrode, located in the immediate vicinity of the sample, makes up with the capacitance included in the oscillating circuit of the high-frequency generator, and serves simultaneously to excite and detect oscillations of the sample. Measurements of the resonance frequency $f_{\mathrm{r}}$ of the oscillation of the sample allow the determination of the elastic modulus $E$ according to:

$$
f_{r}=k \frac{d}{L^{2}} \sqrt{\frac{E}{\rho}}
$$

where $d$ is the thickness of the sample, $L$ is the length of the oscillating plate, $E$ is the modulus of elasticity, $\rho$ is the density of the sample, and $k$ is a constant coefficient. Figure 3 shows the block-holder of the superconducting sample. It is made of ultrapure copper and is placed in a low-temperature cryostat.

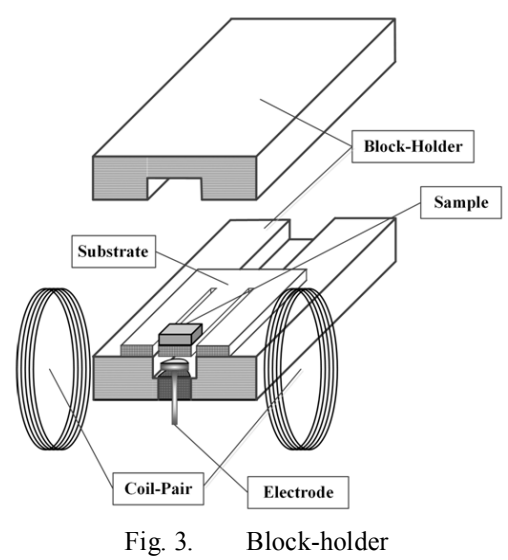

Block-holder provides oscillations of a superconducting sample in constant and alternating magnetic fields without affecting it by mechanical deformations. This is achieved by the fact that the metal substrate acts as a vibrator of a mechanical spectrometer, cut in the shape of E character, and 
the superconducting sample is glued to the tip of its middle reed. As a substrate, high-purity niobium is specially selected. In the temperature range from nitrogen to $200 \mathrm{~K}$, which is of interest to us, there are no anomalies of the elastic modulus and a very low level of oscillation energy dissipation $\left(\sim 5 \times 10^{-4}\right)$. Figure 4 shows a cryostat placed in the gap of a permanent magnet and operating in the temperature range from $80 \mathrm{~K}$ to 500K. In Figure 4: 1) The block-holder with the sample is rigidly fixed on the console of ultrapure copper. 2) The tank into which liquid nitrogen is poured is integrated with the console. 3) A furnace is wound in the lower part of the console. These allow to measure both the temperature spectra and the field dependences of the elastic modulus in the temperature range of $80-500 \mathrm{~K}$. 4) The poles of the permanent magnet are located on both sides of the cryostat and can be moved so that the direction of the constant field $H$ relative to the sample plane can be changed at any angle.

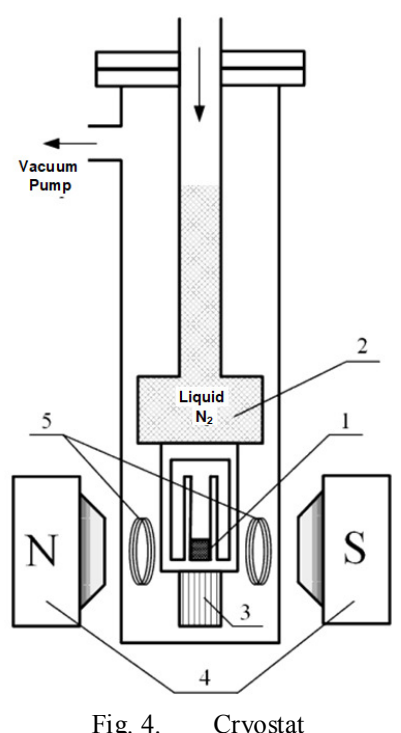

To study the dynamics of the Abrikosov vortex, a lattice in HTSC is provided for the creation of a relatively weak exciting magnetic field, oscillating with a certain frequency. In order to create such a field, 5 especially wound Helmholtz coils are placed inside the cryostat, covering the block-holder. The location of the coils in the immediate vicinity of the sample allows the creation of a sufficiently strong alternating field on it by using a standard low-frequency generator. The alternating field $h$ created by the Helmholtz coils can also be directed at different angles that were combined with application of solar energy and superfast quenching melt technology [1-5]. Amorphous precursors and their use make possible the manufacturing of high-density low porous texturized ceramics with given dimensions of grains. Starting mixtures of nominal composition were prepared from previously annealed $\mathrm{Bi}_{2} \mathrm{O}_{3}$, $\mathrm{PbO}, \mathrm{SrCO}_{3}, \mathrm{CaO}, \mathrm{CuO}$ high purity powders. The synthesis by solar melting and the following superfast quenching was realized using as a heating source the concentrated solar flux in a solar furnace. The phase composition of samples was determined by X-ray phase analysis. The form of superconducting samples used in the experiments was such that their size in the direction of the magnetic field was less than the depth of its penetration. In this case, the Abrikosov magnetic vortices (AV) penetrated the sample through in the direction of the magnetic field.

The variation of the square of the resonant oscillation frequency $\left(f^{2}\right)$ of the sample can be considered in the framework of the so-called magnetomechanical approach [7] according to which, when the superconductor is displaced relative to the external magnetic field $H$, acts as a restoring mechanical force on each "pinned" magnetic vortex. As a result, the oscillation frequency of the entire sample changes by $\Delta f(H)$, depending on the density of the fixed vortices, the moment of inertia of the superconductor and its volume. Measuring the dependence of the elastic modulus of the substrate-sample system (in units of $f^{2}$ ) on the magnitude of the magnetic field gives information about the elastic interaction of the AV with the crystal lattice. This is a convenient method for determining the magnitude of the vortex pinning force, especially by measuring the Labusch parameter, which determines the slope of the pinning potential curve. In addition, the dependence $f^{2}(H)$ provides a simple and convenient method for determining the value of the lower critical magnetic field $H_{\mathrm{c} 1}$. Figure 5 shows the dependence of $f^{2}(H)$ of sample (2267), taken at $T=88 \mathrm{~K}$, while its superconducting transition temperature is $T_{\mathrm{c}}=97 \mathrm{~K}$.

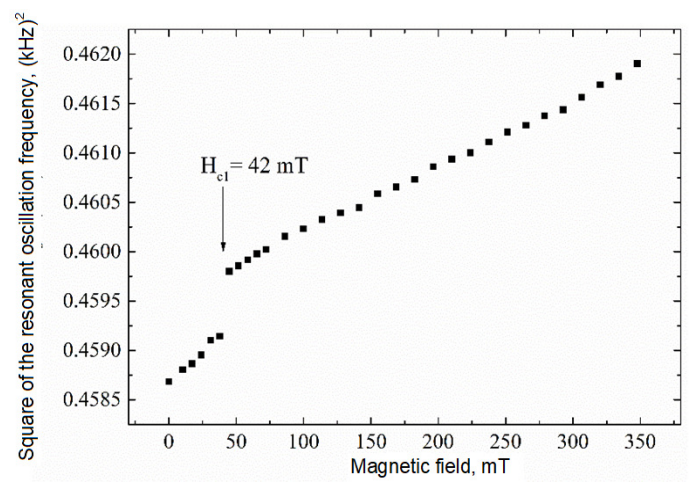

Fig. 5. Field dependence of the square of the natural frequency of the sample (2267)

The anomaly $f^{2}(H)$ at $42 \mathrm{mT}$ shows the moment of the magnetic field vortex penetration into the sample, i.e. $H_{\mathrm{c} 1}$. An increase in $f^{2}$ corresponds to an increase in the number of AVs with an increase in $H$. By the slope of this curve, one can judge the pinning force in this particular sample. One could also study the phase transitions in the Abrikosov vortex lattice. As an example, Figure 6 shows the field dependences of a singlephase polycrystalline sample of $\mathrm{ErBa}_{2} \mathrm{Cu}_{3} \mathrm{O}_{7-\delta}$ prepared according to standard technology. According to the temperature dependence of the electrical resistance $\Omega$ (Figure 6 ), the temperature and width of the superconducting transition were determined: $T_{\mathrm{c}}=91.2 \mathrm{~K}$ and $\Delta T_{\mathrm{c}}=0.8 \mathrm{~K}$ respectively. The dependences of $f^{2}(H)$ were measured in the superconducting state of the sample at $T=79 \mathrm{~K}$. Two different dependencies were taken. In the first case, the sample oscillated only in an external constant magnetic field, (curve $\square$ ). In the second case, along with the constant magnetic field, from the very beginning the 
sample was affected by a weak, $h=0.5 \mathrm{mT}$ alternating magnetic field of low frequency (curve a). On the first curve ( $\square$ ) of the dependence $f^{2}(H)$, at $H=173 \mathrm{mT}$, there is a feature characteristic of order-disorder phase transformations. This feature indicates that when $H=173 \mathrm{mT}$ and higher, AV exist in the sample in the form of ordered lattice. If during the taking of the field dependence a variable magnetic field $h$ acts on the sample, then the feature on the $f^{2}(H)$ curve is not observed. This is due to the fact that the alternating magnetic field constantly shakes the vortices in the sample, preventing their ordering in the lattice. In this work, the temperature dependence of the square of the natural frequency $f^{2}(H)$ of an HTSC sample oscillating in a magnetic field is used to obtain information about the phase composition of high-temperature superconducting precursors above $T_{\mathrm{c}}$. When a superconducting VR is subjected to applied fields and temperature variations, a step-like increase in the resonant frequency $\Delta f=f-f_{\mathrm{o}}$ compared to its normal state value $f_{\mathrm{o}}$ is observed near or below the upper critical magnetic field $H_{\mathrm{c} 2}(T)$, when flux lines are rigidly pinned to VR. This is due to the diamagnetic surface currents flowing in the superconductor and producing diamagnetic restoring force proportional to the $\boldsymbol{M} \times \boldsymbol{H}$ torque. This feature when applied above $T_{\mathrm{c}}$ completely removes normal-state contributions and thus allows tracing the diamagnetic signal above $T_{\mathrm{c}}$ with great precision. Prior to the measurements, the samples were cooled to a nitrogen temperature in an external magnetic field, after which the temperature dependences of $f^{2}$ were taken during a slow, $\approx 1 \mathrm{~K} / \mathrm{min}$, heating. In order to separate the effects associated with the penetration of AV into the superconducting sample from the effects of the niobium substrate proper, we measured the temperature dependence of $f^{2}$ of pure niobium. Figure 7 shows the dependence of $f^{2}(T)$ of a niobium substrate without an HTSC sample taken in an $300 \mathrm{mT}$ external magnetic field.

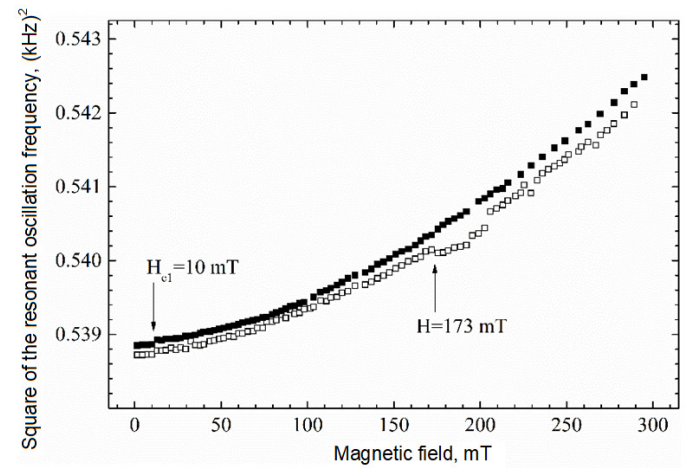

Fig. 6. Field dependence of $f^{2}$ of oscillation of the sample $\mathrm{ErBa}_{2} \mathrm{Cu}_{3} \mathrm{O}_{7-\delta}$ : $\square-h=0, \boldsymbol{\square}-h=0.5 \mathrm{mT} . T=79 \mathrm{~K}$

It is seen that the $f^{2}(T)$ curve, in the temperature range from nitrogen to $170 \mathrm{~K}$, has a uniform slope and does not contain any features. Figure 8 shows the dependence of $f^{2}(T)$ of sample (2267) taken in an external magnetic field of $300 \mathrm{mT}$. The curve contains a single feature at $T=97 \mathrm{~K}$. Such features on the $f^{2}(T)$ dependence are typical for phase transition points, the $97 \mathrm{~K}$ point is the sample transition temperature from normal to superconducting state. There are no other features on the curve, this means that the sample (2267) is monophase. Another picture is observed on the $f^{2}(T)$ curve of the sample (2-2-19-
20), shown in Figure 9. On dependencies taken at a magnetic field of $300 \mathrm{mT}$ during the heating process, 4 features were observed. This means that in the sample (2-2-19-20), along with the main superconducting phase and the transition temperature of $104 \mathrm{~K}$, there are inclusions of other superconducting phases with higher transition temperatures: 118,127 and $151 \mathrm{~K}$.

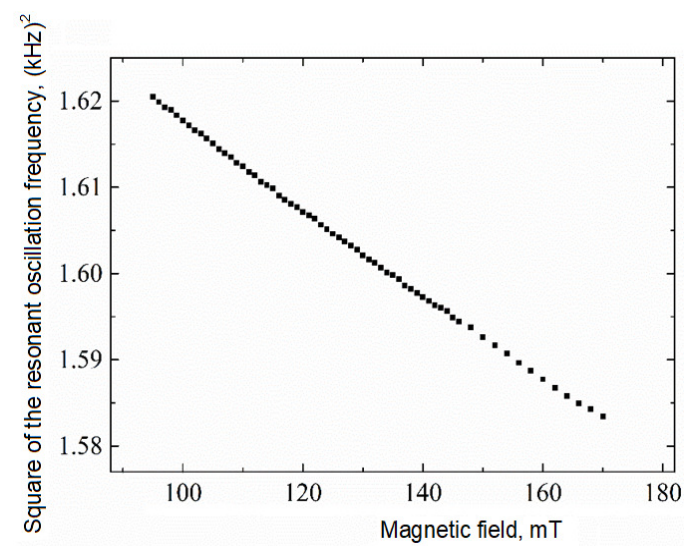

Fig. 7. Temperature dependence of $f^{2}$ of a niobium substrate in a magnetic field of $300 \mathrm{mT}$.

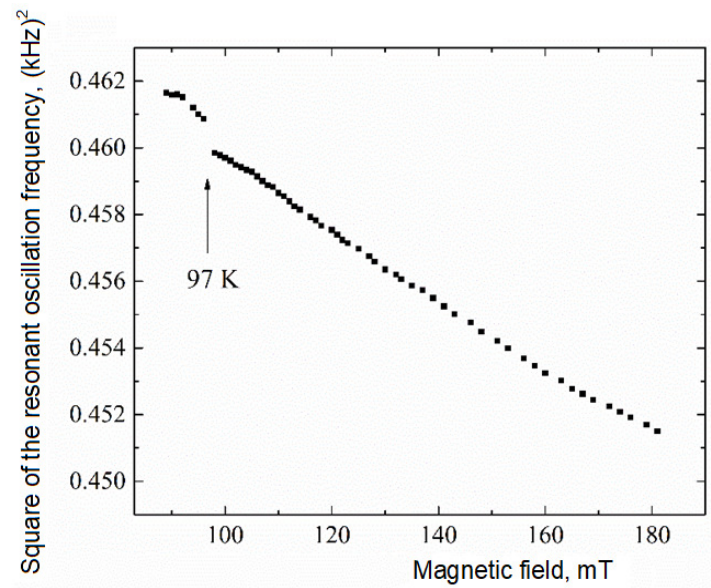

Fig. 8. Temperature dependence $f^{2}$ of oscillations of the monophase sample (2267) in a magnetic field of $300 \mathrm{mT}$

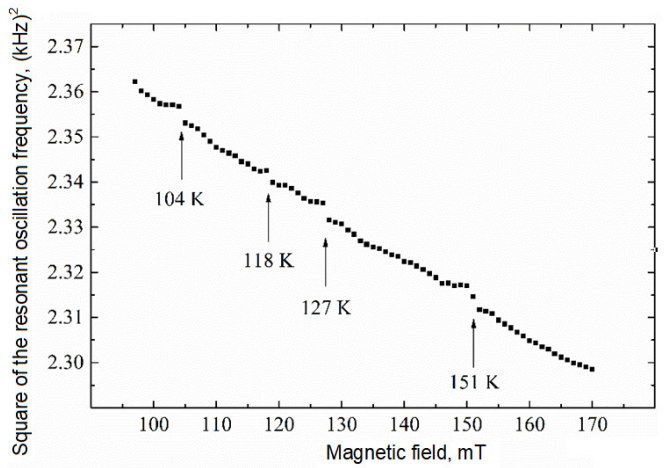

Fig. 9. Temperature dependence of $f^{2}$ of the multiphase sample (2-2-19-20) in a magnetic field of $300 \mathrm{mT}$ 


\section{CONCLUSIONS}

We investigated the potential possibilities of the vibrating reed method for the evaluation of $T_{\mathrm{c}}$ of superconducting precursors in an HTSC Bi-Pb-Sr-Cu-O system. We applied a special technology for obtaining these samples using solar energy for their melting and subsequent uperfast melt quenching for increasing their internal inhomogeneity, allowing the receiving of high-quality textural ceramic samples of the $\mathrm{Bi}_{1,7} \mathrm{~Pb}_{0,3} \mathrm{Sr}_{2} \mathrm{Ca}_{\mathrm{n}-1} \mathrm{Cu}_{\mathrm{n}} \mathrm{O}_{\mathrm{y}}(\mathrm{n}=2-30)$ system showing critical temperatures of superconducting precursor transitions above bulk $T_{\mathrm{c}}$. To determine the critical temperatures $T_{\mathrm{c}}$ of superconducting precursors above bulk $T_{\mathrm{c}}$ we used for the first time the original vibrating reed method to study this multiphase samples in an applied magnetic field. It was shown that this method has sensitivity to superconducting diamagnetism making possible to reveal new superconducting precursor phases above bulk $T_{\mathrm{c}}$.

\section{ACKNOWLEDGMENT}

This work has been supported by the Shota Rustaveli National Science Foundation Grant STCU-2016-51 \& STCU Grant N 6317.

\section{REFERENCES}

[1] J. G. Chigvinadze, J. V. Acrivos, S. M. Ashimov, D. D. Gulamova, T. V. Machaidze, D. Uskenbaev, "Temperature dependence investigation of dissipation processes in strongly anisotropic high-temperature superconductors of $\mathrm{Bi}-\mathrm{Pb}-\mathrm{Sr}-\mathrm{Ca}-\mathrm{Cu}-\mathrm{O}$ system synthesized using solar energy", Epitoanyag, Vol. 61,pp. 104-108, 2009

[2] D. D. Gulamova, D. G. Chigvinadze, J. V. Acrivos, D. E. Uskenbaev, "Obtaining and studying the properties of high-temperature superconductors of homologous series of $\mathrm{Bi}_{1.7} \mathrm{~Pb}_{0.3} \mathrm{Sr}_{2} \mathrm{Ca}_{\mathrm{n}-1} \mathrm{Cu}_{\mathrm{n}} \mathrm{O}_{\mathrm{y}}(n=4-$ 9), under influence of solar energy", Applied Solar Energy, Vol. 48, pp. 135-139, 2012

[3] J. G. Chigvinadze, J. V. Acrivos, S. M. Ashimov, D. D. Gulamova, G. Donadze, "Superconductivity at $T \approx 200 \mathrm{~K}$ in bismuth cuprates synthesized using solar energy", available at: https://arxiv.org/ftp/arxiv/papers/1710/1710.10430.pdf, 2017

[4] J. Chigvinadze, S. Ashimov, G. Mamniashvili, G. Donadze A. Peikrishvili, B. Godibadze, "On the Nature of Superconducting Precursors in $\mathrm{Bi}-\mathrm{Pb}-\mathrm{SrCa}-\mathrm{Cu}-\mathrm{O}$ Compositions Fabricated by Hot Shock Wave Consolidation Technology", Engineering, Technology \& Applied Science Research, Vol. 8, No. 3, pp. 3032-3037, 2018

[5] J. G. Chigvinadze, S. M. Ashimov, J. V. Acrivos, D. D. Gulamova, "Critical temperature of the superconducting transition of individual phases of multiphase bismuth cuprates after cooling in a magnetic field to a temperature of 77 K", Low Temperature Physics, Vol. 45, No. 4. pp. 386-394, 2019

[6] S. M. Ashimov, D. G. Chigvinadze, "Torsion balance for studying anisotropic magnetic properties of superconducting materials", Instruments and Experimental Techniques, Vol. 45, No. 5, pp. 431-435, 2002

[7] P. Esquinazi, "Vibrating superconductors", Journal Low Temperature Physics, Vol. 85, No. 3-4, pp. 139-232, 1991

[8] A. Gupta, P. Esquinazi, H. F. Braun, "Vibrating-reed studies on nonsingle-phase Bi-Ca-Sr-Cu-O superconducting ceramics", Physical Review B, Condensed Matter, Vol. 39, No. 16, pp. 12271-12274, 1989

[9] V. A. Melik-Shakhnazarov, I. A. Naskidashvili, "A device for an automated recording of internal friction in metals", Pribory i Tekhnika Eksperimenta, Vol. 1, pp. 81-84, 1967 (in Russian) 